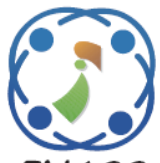

\title{
Copula-Monte Carlo Based Probabilistic Oscillatory Stability Analysis of Microgrid
}

\author{
Awan Uji Krismanto ${ }^{1}$ \\ N Mithulananthan ${ }^{2}$ \\ Abraham Lomi ${ }^{1}$ \\ Muhammad Abdillah ${ }^{4}$ \\ Herlambang Setiadi ${ }^{3 *}$ \\ ${ }^{1}$ Department of Electrical Engineering, Faculty of Industrial Technology, \\ Institut Teknologi Nasional Malang, Indonesia \\ ${ }^{2}$ School of Information Technology and Electrical Engineering, The University of Queensland, Brisbane, Australia \\ ${ }^{3}$ Faculty of Advanced Technology and Multidiscipline, Universitas Airlangga, Surabaya, Indonesia \\ ${ }^{4}$ Department of Electrical Engineering, Universitas Pertamina, Indonesia \\ * Corresponding author’s Email: h.setiadi@stmm.unair.ac.id
}

\begin{abstract}
This paper investigates effects of wind speed and solar irradiance uncertainties on oscillatory stability of Microgrid (MG). Novel estimation technique based on Student's $t$-Copula is applied to estimate the probability distribution function $(P D F)$ of wind speed and solar irradiance by taking into account the stochastic dependencies between those energy resources. A detailed MG system comprising of Wind Energy Conversion System (WECS), PV system and Diesel Engine (DE) based distributed generation (DG) units is considered to provide a complete dynamic response of the investigated MG. Distribution of critical modes damping ratio is thoroughly investigated by means of Monte Carlo Simulation (MCS). Probabilistic study of small signal stability in MG implies that the renewable energy resources (RES) uncertainties result in a dynamic change of power-sharing strategies and introduce adverse effects on small signal stability. In addition, the damping value is variated from critical damped (with probability around 0.004), well damped and under damped. Moreover, it was monitored that wind speed fluctuation brings more severe impact on system damping than irradiance variation. Consequently, the MG experienced more oscillatory conditions and even lead to unstable situation at high wind speed circumstances. While under solar irradiance change, the investigated MG system can maintain its stable operation.
\end{abstract}

Keywords: Copula, Microgrid, Monte-carlo, Renewable energy, Oscillatory stability, Uncertainties.

\section{Introduction}

A cluster of RES based DG units such as WECS, $\mathrm{PV}$ and its combinations might be operated into a single coordinated and controlled power system namely as microgrid (MG). The MG system mostly operated in islanding mode due to the absence or limitation of distribution line provided by the utilities. When operated independently from the existing grid, MG should be able to provide an accurate powersharing scheme among DGs while maintaining the stability of local voltage and frequency. Moreover, uncertain RES condition such as wind speed and solar irradiance variations introduced novel challenges to MG operation, involving small signal stability. As wind speed and solar irradiance varied, power output from the corresponded DGs fluctuated accordingly. Power sharing scheme should be adjusted continuously to manage with random RES change or variation. Change of power-sharing might bring adverse effect on system dynamic response [1-3]. Oscillatory stability concern potentially emerges due to change of $\mathrm{MG}$ operating point after being subjected to a small perturbation in the power-sharing scheme.

Since the RES uncertainties significantly affect the contribution of each DGs in MG, risk assessment and small signal stability analysis of islanding MG operation is crucial. Comprehensive small signal stability study involving all possible conditions of RES should be considered to evaluate dynamic 
performance and determine the system stability boundaries. The conventional method to analyse the oscillatory stability is the modal analysis based on a deterministic approach. However, the main consideration of deterministic analysis is the incapability to capture random or stochastic behaviours due to uncertainties $[4,5]$. Since the RES varying continuously, the system operating point would be stochastically uncertain. As a consequence, it is difficult to obtain a complete picture regarding the risk of instability and the distribution of possible system operating point using the deterministic approach [6]. This limitation motivated the development of novel approach in analysing oscillatory stability considering uncertainties. Monte Carlo Simulation (MCS) has been a popular method for solving a probabilistic oscillatory stability problem in power system. Although MCS required a high computational time, it provides an accurate result and flexibility to handle a large number of data samples compared with another analytical method [7]. Implementation of MCS to investigate the impact of wind generation uncertainty on angle stability has been investigated in [8-10]. In [8], it was found that, representing intermittent wind speed by using probabilistic approach has significant influence of small signal stability. Research effort in [9], investigate the application of monte carlo simulation for modelling the uncertainty of wind speed. In addition, research effort in [10] shows that the impact of integrating probabilistic wind power system can be handled by adding SVC and PSSs. It was observed that an adverse effect on small signal stability was monitored as power injection from WCES increase. Dynamic behaviour of PV-Diesel MG under the uncertain condition of solar irradiance and load was investigated in $[11,12]$.

The accuracy of MCS is highly dependent on the estimation of random variable probabilistic distribution functions $(P D F s)$. The distribution functions of RES such as wind speed and solar irradiance are difficult to be accurately estimated since they are strongly dependent on ambient temperature, climate, and geographical positions [13]. Many studies have been developed for modelling the distribution function of RES for stability analysis purpose [4-6]. However, those previous studies did not consider dependencies among varying variables. Since both of wind speed and solar irradiance are influenced by environmental circumstances, it is important to take into account the potential correlation between variability of wind speed and solar irradiance in modelling their PDFs. In [14, 15], the correlation and analysis of the climatological relationship between wind and solar energy are presented. It was monitored that at certain areas, there is a complementary relationship between wind speed and solar irradiance.

To take into account the correlation or dependence in RES modelling, a copula estimation technique is proposed. Copula method is a popular method to generate a multivariate distribution function from their marginal univariate distribution and provide a correlation structure between random variables. Moreover, the proposed technique is feasible to estimate the PDFs of stochastic variables which have distributions, not from a standard multivariate distribution. A copula method for estimating correlation of instantaneous solar irradiance in the spatial network is presented in [16]. Estimation of wind speed and solar irradiance cumulative distribution functions (CDFs), considering their dependencies, using a diagonal band copula is presented in [13]. Moreover, the stochastic correlation among wind power generator in a certain area was modelled using Gaussian copula as presented in [17].

Even though the dependence of RES might influence the dynamic behaviour of MG, only a few works considering the RES correlation in the probabilistic small signal stability analysis were reported. This paper presents an analysis of oscillatory stability performance in islanding MG operation considering the dependence model of wind speed and solar irradiance. A copula estimation technique is adopted in this paper to generate more practical PDFs of wind speed and solar irradiance scenarios. The determined stochastic RES model is applied to hybrid MG system comprising of WECS, PV and diesel engine (DE) based DG units. Dynamic response, damping performance and oscillatory frequency of the hybrid MG under the stochastic condition of RES are observed through MCS. The eigenvalues obtained from the MCS are clustered using a spectral clustering technique to extract the critical modes. Risk of instability and small signal stability performance of the investigated MG system are then evaluated based on statistical properties of the critical eigenvalues.

The remainder of the paper is organized as follows. Estimation procedure of the wind speed and solar irradiance using copula approach and MCS of probability small signal stability are presented in Section II. In Section III, a detailed modelling procedure of hybrid MG involving dynamic models of the control system, distribution network and load are presented. The simulation results are presented and discussed in Section IV. Eventually, conclusions and contributions of this paper are highlighted in Section V. 


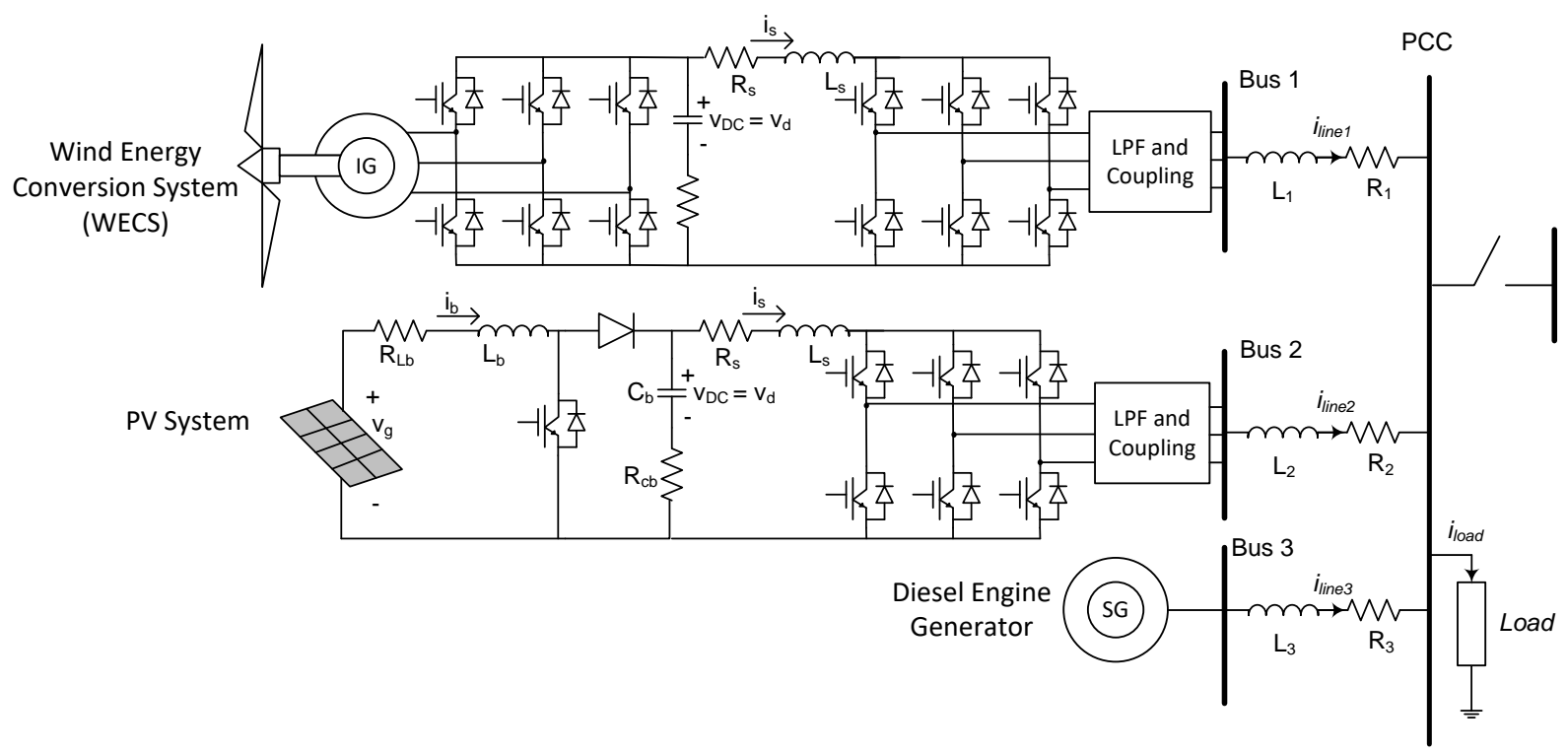

Figure. 1 Investigated microgrid model

\section{Microgrid model}

Fig. 1 depicts the investigated hybrid Microgrid (MG) system comprising of Wind-PV and Diesel Engine (DE) based DG units. Full Converter WECS incorporates back-to-back AC/DC/AC converter as interfacing device between the wind turbine and local bus. While in two stages PV system, boost DC/DC and DC/AC inverter system facilitated the connection between solar PV array and local bus. Moreover, high order component due to high frequency switching features of power electronic devices in RES based DGs is handled by one order L-C-L low pass filter.

It was clearly reported in $[18,19]$ that $\mathrm{MG}$ dynamic behaviour is strongly influenced by power contributions of each DG units within MG. The contributions of RES based DG units are determined through power sharing control scheme which are realized using droop control method. In order to ensure a balance condition between generated and demanded power, droop parameters of WECS and PV based DG units should be updated continuously in proportion with wind speed and solar irradiance changes. In this research, a modified droop method is proposed to facilitate an adaptive power sharing scheme from each RES based DG unit. The proposed control strategy is then applied in grid-side converter controller of WECS and PV based DG unit as depicted in Fig. 2.

As depicted in Fig. 2, the grid side inverter control involves a modified droop control strategy, voltage and current control loops. The modified droop control adjusts droop parameter adaptively to provide a precise power sharing scheme among DG units when the MG is operated under fluctuated condition of wind speed and solar irradiance. Calculated reference values from the modified droop control are then applied to voltage and current control loops. Voltage control loop enhances the system dynamic response while current control loop is responsible for generating a modulation signal for DC/AC inverter.

Average active $\left(\Delta P_{\text {meas }}\right)$ and reactive $\left(\Delta Q_{\text {meas }}\right)$ power are determined by filtering the instantaneous active and reactive power as given by

$$
\begin{aligned}
& \Delta P_{\text {meas }}=\left\{\frac{\omega_{c}}{\left(s+\omega_{c}\right)}\right\} p \\
& \frac{d \Delta P_{\text {meas }}}{d t}=\omega_{c} p-\omega_{c} \Delta P_{\text {meas }} \\
& \Delta Q_{\text {meas }}=\left\{\frac{\omega_{c}}{\left(s+\omega_{c}\right)}\right\} q \\
& \frac{d \Delta Q_{\text {meas }}}{d t}=\omega_{c} p-\omega_{c} \Delta Q_{\text {meas }}
\end{aligned}
$$

Reference values of frequency $\left(\omega^{*}\right)$ and voltage $\left(v^{*}\right)$ are determined as a function of droop parameter according to the following equations

$$
\begin{aligned}
\omega^{*} & =\omega_{n}-m_{D G-v a r}\left(\Delta P_{\text {in }}-\Delta P_{\text {meas }}\right) \\
v_{o d}^{*} & =v_{n}-n_{q}\left(\Delta Q_{\text {in }}-\Delta Q_{\text {meas }}\right)
\end{aligned}
$$

Active and reactive droop gains selection are conducted as a compromise between accurate powersharing, enhancement of dynamic response and 


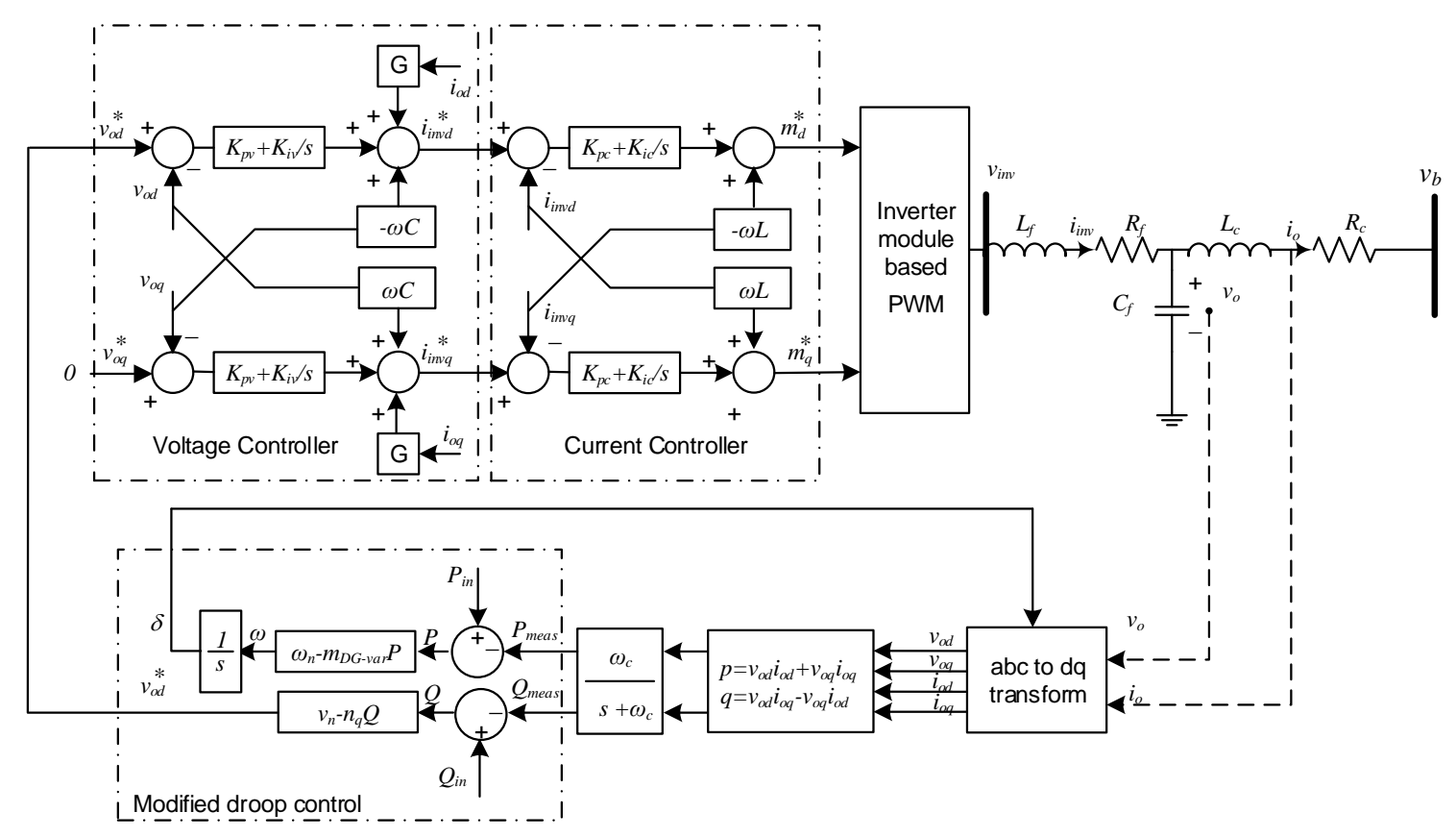

Figure. 2 Modified droop control method

voltage regulation. In this study, only active power variation from RES is considered since MG small signal stability less sensitive to reactive droop gain variation [1-3]. Reactive power droop gains in each DGs are maintained constant throughout this work. RES uncertainties in terms of fluctuating conditions of solar irradiance and wind speed significantly influence the power-sharing scheme among DG units. To handle RES uncertainties, the droop gain values should be adjusted continuously to ensure accurate power sharing in accordance with RES variations. Moreover, during islanding operation, each of DGs should contribute to maintaining the system frequency. The frequency support from each DGs is feasible if there is sufficient power margin. Hence, to ensure adequate power preserve for frequency regulation purpose, the WECS and PVs might be operated slightly below its maximum operating point. A proposed dynamic droop parameter considering RES variation and power preserve for frequency regulation purpose is given by

$m_{D G-v a r}=\left(m_{\max }-\left(m_{\max }-m_{\min }\right)\left\{\frac{P_{\text {input }}}{P_{\max }}\right\}\right)$

Where $P_{\text {input }}$ and $P_{\max }$ represent actual input power from RES and maximum power of DGs respectively.

The active droop gain control is regulated within the specified maximum $\left(m_{\max }\right)$ and minimum $\left(m_{\min }\right)$ limit.

For PV based DG, the droop gain can be calculated by substituting generated input power from PV as a function of solar irradiance [20] to Eq. (10) as given by

$m_{P V-v a r}=\left(\begin{array}{c}m_{\max }-\left(m_{\max }-m_{\min }\right) \times \\ \frac{V_{P V} N_{P}}{P_{\max }} \times\left\{I_{s c}\left(\frac{G_{0}}{G_{\text {ref }}}\right)-I_{s} e^{\frac{q V_{P V}}{n k T N_{s}}}\right\}\end{array}\right)$

Where $G_{r e f}$ and $G_{0}$ represent a reference and initial values of solar irradiance around a certain operating point respectively. Number of parallel and series solar panel are represented by $N_{p}$ and $N_{s}$ respectively. Electrical variables of PV arrays which represent short circuit current and voltage of PV array are represented by $I_{S C}$ and $V_{P V}$ respectively. While, $T$ shows an ambient temperature. $n$ and $k$ represent constants values of PV arrays.

Similarly, for wind based DG unit, the droop gain can be calculated by substituting generated input power from WECS as a function of actual wind speed [21] to Eq. (10) as given by

$$
m_{W E C S-v a r}=\left(\begin{array}{c}
m_{\max }-\left(m_{\max }-m_{\min }\right) \times \\
\left\{\frac{0.5 \rho C_{\text {opt }}\left(\lambda_{\text {opt }}, \beta\right) A_{r} v_{w 0}^{3}}{P_{\max }}\right\}
\end{array}\right)
$$


Where $v_{w 0}$ represents an initial condition of wind speed around a certain operating point. It is assumed that power input from a certain wind speed is a function of a given wind speed with constant tip speed ratio and blade pitch angle. $C_{o p t}$ represents an efficiency constant of wind turbine.

Development procedure of state space model of the investigated MG system, comprising of WECS, PV and Diesel engine based DG units, is represented in $[18,19]$. The complete model of the hybrid MG system incorporates state space model of solar array, wind turbine, electrical machines, power electronic devices, controller, distribution network and lumped load. The dynamic behaviour of the MG system which is refleted in variation of critical eigenvalues, is then investigated under uncertain condition of wind speed and solar irradiance.

\section{Method}

\subsection{Copula for wind speed and solar irradiance estimations}

Annual historical data of wind speed with threehour measurement resolutions and an hourly solar irradiance between 2014-2015 from two weather stations in Rockhampton, Queensland, Australia are considered. The collected data are seasonally clustered. The seasonal data are then further divided into 24 hourly data segments. For small signal stability analysis of islanding MG operation, it is assumed that all DG units in MG participate in supplying load demand. Hence, the only dynamic behavior of hybrid MG during daytime is considered. Thus, there are 4 combinations of wind speed and solar irradiance data for the year in each location. Considering a month is equivalent to 30 days, each season time segment comprising of 90 wind speed and solar irradiance data.

The selected hourly data of wind speed and solar irradiance are applied to copula function to generate stochastic distribution variables for MCS. The copula is a function that links or join multivariate distribution functions to their one-dimensional marginal distribution functions [22]. Derived from Sklar's theorem, the random variables of $\boldsymbol{X}$ and $\boldsymbol{Y}$ with their CDF of $F_{X}$ and $F_{Y}$ respectively can be linked by a copula function $C$ as given by

$$
F_{X Y}(x, y)=C\left(F_{x}(x), F_{y}(y)\right)
$$

Multivariate distribution function in a copula can be presented as a uniform distribution by transforming the $\mathrm{CDF}$ of an arbitrary random variable $\boldsymbol{X}$ and $\boldsymbol{Y}$. Consequently, a uniform variables distribution can be transformed into its actual values by using the inverse of $C D F(i C D F)$. Transformation of actual random variables of $\boldsymbol{X}$ and $\boldsymbol{Y}$ values into the uniform distributions on the interval $[0,1]$ can be stated as $F_{x}(x)=u$ and $F_{y}(y)=v$ respectively. By applying $i C D F$ to the uniform values, the copula function of the actual values of $u$ and $v$ is given by

$C_{U V}(u, v)=F\left(F_{x}^{-1}(u), F_{y}^{-1}(v)\right)$

Students' $t$ copula is employed in this study to provide a stochastic model of wind speed and solar irradiance considering possible dependence between those RES. The $t$-copula has been selected due to superior features comparing with the Gaussian copula. Moreover, it had the better feasibility to capture the extreme values of random variables [23]. In simulating $t$-copula, a multivariate $t$-distributed random vector $\boldsymbol{X} \sim t_{d}(v, \boldsymbol{\mu}, \Sigma)$ is generated according to the density function as given by the following equation

$f(x)=\frac{\Gamma\left(\frac{v+d}{2}\right)}{\Gamma\left(\frac{v}{2}\right) \sqrt{(\pi v)^{2}|\Sigma|}} \times\left(1+\frac{(x-\mu)^{\prime} \Sigma^{-1}(x-\mu)}{v}\right)$

Where $v$ is degrees of freedom, $\mu$ is mean of vector random variables and $\Sigma$ represents positive-definite dispersion or scatter matrix.

Under normalization of the marginal distribution, the $t$-copula remains invariant, hence it can be considered that the copula of $\boldsymbol{X} \sim t_{d}(v, \boldsymbol{\mu}, \Sigma)$ is identical to $\boldsymbol{X} \sim t_{d}(v, \mathbf{0}, P)$. Where $P$ represents the correlation-matrix implied by the dispersion of matrix $\Sigma$. For estimating purposes, the density of $t$ copula might be determined from Eq. (2) and given by the following equation [23].

$C_{v, P}^{t}(\mathbf{u})=\frac{f_{v, P}\left(t_{V}^{-1}\left(u_{1}\right), \ldots, t_{V}^{-1}\left(u_{d}\right)\right)}{\Pi_{i=1}^{d} f_{v}\left(t_{V}^{-1}\left(u_{i}\right)\right)}, \mathbf{u} \in(0,1)^{d}$

Where $f_{v, P}$ is the joint density of a $\boldsymbol{X} \sim t_{d}(v, \mathbf{0}, P)$ and $f_{v}$ represents the density of univariate standard $t$ distribution with $v$ degrees of freedom.

The strength of dependence between random variables can be measured through rank correlation $\left(\rho_{r}\right)$ values. The rank correlation of random variables $\boldsymbol{X}$ and $\boldsymbol{Y}$ with CDFs of $F_{X}$ and $F_{Y}$ is defined as [13, 17].

$$
\rho_{r}(x, y)=\rho\left(F_{x}(X), F_{y}(Y)\right)
$$

The rank correlation varies in the range of $[-1,1]$ 
which corresponded to perfect negative and positive dependence respectively. Negative dependence represents the complementary relationship between random variables; increase values of one variable associated to decrease of another random variable. While positive dependence indicated a monotonic relationship between variables to which large or small values of one variable related to large or small values of other variables. The product moment correlation of stochastic variables is given by the following equation [17].

$\rho(X, Y)=2 \sin \left(\frac{\pi}{6} \rho_{r}(X, Y)\right)$

Estimation of $t$-copula parameters $(v$ and $P$ ) can be approached by using maximum likelihood method based on maximization of the joint probability density of random variables as given by

$\log L\left(v, P ; \widehat{\mathbf{U}}_{1}, \ldots, \widehat{\mathbf{U}}_{n}\right)=\sum_{i=1}^{n} \log C_{v, P}^{t}\left(\widehat{\mathbf{U}}_{i}\right)$

Where $C_{v, P}^{t}$ denotes density function of the $t$ copula as stated in Eq. (4).

By applying the inverse of $C D F(C D F)$ to the $t$ copula function as given by Eq. (2), the estimated actual values of a stochastic variable can be determined.

\subsection{Monte carlo simulation for small signal stability}

The influence of uncertain conditions of wind speed and solar irradiance on the dynamic behaviors of hybrid MG system is evaluated through MCS method. The distribution of wind speed and solar irradiance historical data from two weather stations were estimated by means of $t$-copula, considering uncertainties and possible dependencies among RES. The framework of probabilistic small signal stability analysis using MC method is depicted in Fig. 3

The values of wind speed and solar irradiance obtained from joint distribution function from the $t$ copula method are randomly sampled to realize the uncertain condition of hybrid MG operating points. The non-linear dynamic response of critical eigenvalues in different operating points under RES variations are assessed using modal analysis method. Trajectories and damping ratio of critical modes are evaluated to determine the risk of instability and small signal stability performance.

Under uncertainties, sensitive eigenvalues behaved randomly. Hence, it is difficult to observe the dynamic response of the critical modes.

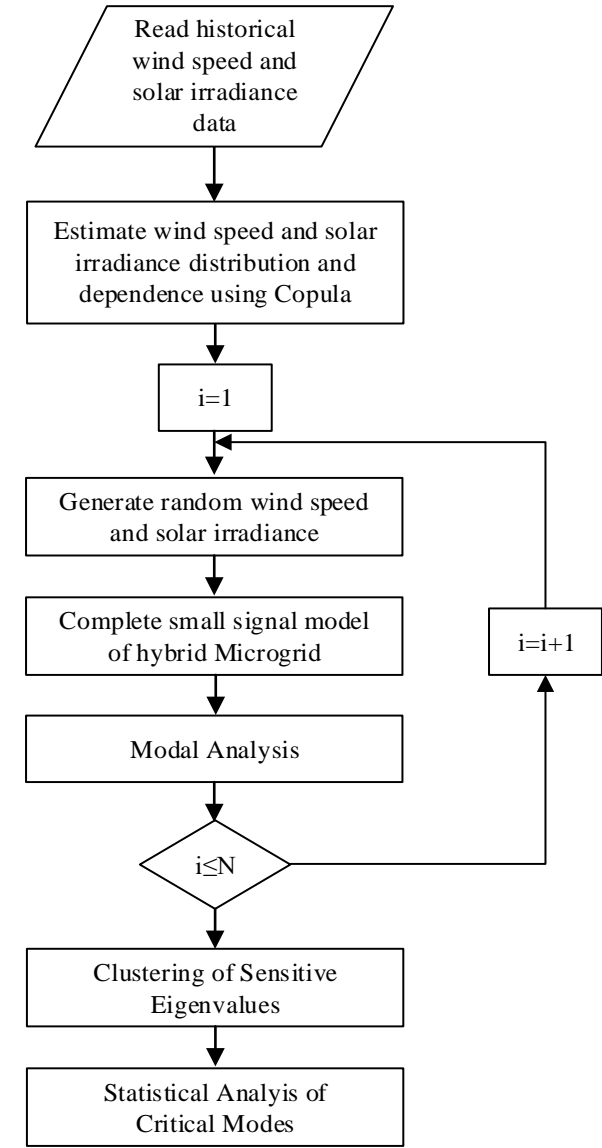

Figure. 3 Monte carlo simulation for probability small signal stability analysis

Conventionally, eigenvalues characterization is performed by monitoring contributions of state variables in those corresponded modes. However, in stochastic small signal stability analysis with a large number of eigenvalues obtained from MCS, it is difficult to specify the eigenvalues. To overcome this problem, the participation factor analysis should be combined with clustering method such as K-means clustering method. In this paper, the K-means clustering is applied to cluster the eigenvalues with an oscillatory frequency less than $2 \mathrm{~Hz}$ since most of the critical eigenvalues which dominantly influence the dynamic response of investigated hybrid MG are characterized by oscillatory frequency below $2 \mathrm{~Hz}$. Euclidean distance among those selected modes is evaluated iteratively to determine the cluster of eigenvalues.

From the clustering stage, the statistical features corresponded to the distribution of real and imaginary parts of critical eigenvalues, system damping and risk of instability are evaluated. The damping ratio $(\zeta)$ of the sensitive modes are characterized into three categories as follows: well-damped condition with $\zeta$ $\geq 5 \%$, critically damped with $5 \% \geq \zeta \geq 0 \%$ and undamped with $\zeta \leq 0 \%$. The well-damped and critically damped categories are related to stable 
system situation and marginally stable situation with more oscillatory conditions or even continuously oscillatory conditions when the system damping ratio is nearly to $0 \%$ respectively. While the undamped situation corresponds unstable situation which typically characterized by growing amplitude of oscillatory condition when the system is subjected to small disturbance.

\section{Results and Discussions}

The small signal model of hybrid MG comprising of 3 MVA WECS, PV and DE generator, as illustrated in fig.3, was investigated. Parameters of the proposed RES based DGs and DE were derived from [18], [19]. Detailed hybrid MG model combined with the state space model of the network impedance to supply aggregated $5 \mathrm{MW}$ central load. Since stability in MG during islanding operation is significantly influenced by dynamic power sharing scheme among DGs, the modal analysis in this study was focused on critical eigenvalues corresponded to the power output of each DGs in MG considering uncertainties of wind speed and solar irradiance. Statistical features of critical eigenvalues related to its damping ratio and oscillatory frequency distributions are thoroughly observed to assess the system dynamic performance and risk of instability events.

\subsection{Wind speed and solar irradiance estimation}

Annual wind speed data and solar irradiation from two different locations in Australia are considered. From collected hourly measurement of RES data, it is difficult to fit the stochastic RES data into a standard distribution function. Moreover, to provide better fitting RES data, possible correlation or dependence between wind speed and solar irradiance should be taken into account in developing proper distribution RES functions. Due to the limitation of the standard functions, the RES estimation can be approached using bivariate distribution function method such as Copula. The student $t$-copula considering the possible dependence and stochastic nature characteristic of RES is employed in the proposed modelling strategy. Obtained fitting data results of wind speed and irradiance from the proposed Copula are compared with the conventional Weibull and Gaussian Mixture distribution functions.

The connection between wind speed and solar irradiation might emerge due to their dependencies to the weather conditions. The rank correlation between seasonal wind speed and solar irradiance in both
Table 1. Rank correlation between wind speed and solar irradiance

\begin{tabular}{ccc}
\hline \hline Seasons & Location 1 & Location 2 \\
\hline \hline Jan-Mar & 0.1297 & 0.0765 \\
Apr-Jun & 0.1991 & 0.3172 \\
Jul-Sep & 0.0684 & 0.1726 \\
Oct-Des & -0.0118 & -0.0146 \\
\hline \hline
\end{tabular}

locations is presented in Table 1. It was monitored that connection between those two stochastic RES variables varied accordingly either in complementary or monotonic trends, indicated by negative and positive rank correlation respectively. In general, a weak dependence between wind speed and solar irradiance existed in both locations, indicated by lowrank correlations varied from -0.0118 to 0.3 . While higher correlations observed during fall season in location 2 as indicated by 0.3172 of rank correlation.

Root Mean Square Error (RMSE) between the CDF of actual and simulated RES values is employed for evaluating the obtained results from Copula and standard distribution methods. The calculation of RMSE is given by the following equation

$R M S E=\sqrt{\frac{1}{N_{d}}} \sum_{j=1}^{N_{d}}\left(C D F_{a c t}^{j}-C D F_{e s t}^{j}\right)^{2}$

Where $N_{d}$ represents number of data sampling. The $C D F_{\text {act }}^{j}$ and $C D F_{\text {est }}^{j}$ respectively corresponded to actual and estimated RES values.

The RMSE values were calculated for each CDFs from $t$-copula, Weibull and Gaussian Mixture Distributions. Table 2 and 3 represents the obtained RMSE values of seasonal wind speed and irradiance CDFs from location 1 and 2 respectively. From the results presented in both tables, it was evident that proposed copula method provides better performance than other standard distribution functions in modelling stochastic variables of wind speed and solar irradiance. Much lower RMSE values from $t$ copula than Weibull and Gaussian Mixture distribution functions were observed. Lower RMS values indicated more accurate data fitting than other technique using standard distribution functions.

Statistical analysis corresponding to the expected values and standard deviation of the estimated wind speed and solar irradiance in location 1 and 2 are presented in Table 4. Higher mean and standard deviation values of wind speed in location 2 than location 1 suggested that the location 2 had more fluctuating wind speed situation throughout the year. Moreover, it was also observed that solar irradiation was relatively similar in the two areas. 
Table 2. Comparison of RMSE for copula, Weibull and gaussian mixture in location 1

\begin{tabular}{|c|c|c|c|c|c|c|c|c|}
\hline \multirow{2}{*}{$\begin{array}{c}\text { Estimation } \\
\text { Method }\end{array}$} & \multicolumn{2}{|c|}{ "Summer } & \multicolumn{2}{|c|}{ Fall } & \multicolumn{2}{|c|}{ Winter } & \multicolumn{2}{|c|}{ Spring } \\
\hline & $\begin{array}{l}\text { Wind } \\
\text { speed }\end{array}$ & $\begin{array}{c}\text { Solar } \\
\text { Irradiance }\end{array}$ & $\begin{array}{l}\text { Wind } \\
\text { speed }\end{array}$ & $\begin{array}{c}\text { Solar } \\
\text { Irradiance }\end{array}$ & $\begin{array}{l}\text { Wind } \\
\text { speed }\end{array}$ & $\begin{array}{c}\text { Solar } \\
\text { Irradiance }\end{array}$ & $\begin{array}{l}\text { Wind } \\
\text { speed }\end{array}$ & $\begin{array}{c}\text { Solar } \\
\text { Irradiance }\end{array}$ \\
\hline$t$-copula & 0.087175 & "0.033221 & 0.056798 & 0.039828 & 0.067773 & 0.048591 & 0.07445 & 0.028225 \\
\hline Weibull & 0.137607 & -- & 0.101921 & -- & 0.087687 & -- & 0.08015 & -- \\
\hline $\begin{array}{l}\text { Gaussian } \\
\text { Mixture }\end{array}$ & -- & 0.145305 & -- & 0.172018 & -- & 0.197105 & -- & 0.309846 \\
\hline
\end{tabular}

Table 3. Comparison of RMSE for copula, Weibull and gaussian mixture in location 2

\begin{tabular}{|c|c|c|c|c|c|c|c|c|}
\hline \multirow[b]{2}{*}{$\begin{array}{c}\text { Estimation } \\
\text { Method }\end{array}$} & \multicolumn{2}{|c|}{ Summer } & \multicolumn{2}{|c|}{ Fall } & \multicolumn{2}{|c|}{ Winter } & \multicolumn{2}{|c|}{ Spring } \\
\hline & $\begin{array}{l}\text { Wind } \\
\text { speed }\end{array}$ & $\begin{array}{c}\text { Solar } \\
\text { Irradiance }\end{array}$ & $\begin{array}{l}\text { Wind } \\
\text { speed }\end{array}$ & $\begin{array}{c}\text { Solar } \\
\text { Irradiance }\end{array}$ & $\begin{array}{l}\text { Wind } \\
\text { speed }\end{array}$ & $\begin{array}{c}\text { Solar } \\
\text { Irradiance }\end{array}$ & $\begin{array}{l}\text { Wind } \\
\text { speed }\end{array}$ & $\begin{array}{c}\text { Solar } \\
\text { Irradiance }\end{array}$ \\
\hline$t$-copula & 0.067951 & 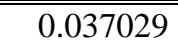 & 0.063574 & 0.043197 & 0.041458 & 0.067203 & 0.056235 & 0.037533 \\
\hline Weibull & 0.166232 & -- & 0.100875 & -- & 0.085699 & -- & 0.109611 & -- \\
\hline $\begin{array}{l}\text { Gaussian } \\
\text { Mixture }\end{array}$ & -- & 0.141647 & -- & 0.174367 & -- & 0.16809 & -- & 0.239005 \\
\hline
\end{tabular}

Table 4. Statistical analysis of four solar irradiance regimes

\begin{tabular}{ccccccccc}
\hline \hline \multirow{2}{*}{ Period } & \multicolumn{4}{c}{ Location 1 } & \multicolumn{4}{c}{ Location 2 } \\
\cline { 2 - 9 } & \multicolumn{2}{c}{ Wind Speed } & \multicolumn{2}{c}{ Solar Irradiance } & \multicolumn{2}{c}{ Wind Speed } & \multicolumn{2}{c}{ Solar Irradiance } \\
\cline { 2 - 9 } & Mean & Std. & Mean & Std. & Mean & Std. & Mean & Std. \\
\hline \hline Jan-Mar & 7.635 & 3.481 & 790.029 & 285.271 & 9.752 & 3.132 & 809.398 & 270.201 \\
Apr-Jun & 8.111 & 2.418 & 628.550 & 191.848 & 10.043 & 3.170 & 633.202 & 211.635 \\
Jul-Sep & 6.515 & 2.503 & 704.904 & 216.303 & 9.788 & 3.051 & 707.772 & 169.767 \\
Oct-Des & 9.378 & 3.201 & 912.545 & 191.313 & 11.348 & 2.748 & 874.148 & 224.889 \\
\hline
\end{tabular}

\subsection{Probabilistic small signal stability analysis considering RES uncertainties}

Stochastic data from $t$-copula technique were applied to investigate dynamic behaviour of the MG system under uncertain conditions of wind speed and irradiance. During autonomous mode, all DG unit in MG participated in the power-sharing scheme. To investigate effects of uncertain RES condition on the oscillatory and dynamic response of $\mathrm{MG}$, it was assumed that WECS and PV based DGs dominantly contributed in supplying power for the load demand. While DE was operated as a reference DG unit which provides a reference synchronization signal and overcomes the shortfall power from those two RES based DG units.

Power contribution of WECS and PV based DGs strongly correlated to the wind speed and solar irradiance conditions respectively and setting of droop controller. Hence, the droop gain should be adjusted continuously within its maximum and minimum setting to manage with RES variation. In this paper, a maximum and minimum active power droop gain were set at $8 \%$ and $4 \%$ respectively. As wind speed and solar irradiance fluctuated, the droop gain would vary accordingly, result in a change of operating points and power contributions of each DG
Table 5. Investigated critical modes

\begin{tabular}{|c|c|c|c|}
\hline Eigenvalue & $\begin{array}{c}\text { Complex } \\
\text { values }\end{array}$ & $\begin{array}{c}\text { Damping } \\
(\%)\end{array}$ & $\begin{array}{c}\text { Participation } \\
\text { Factor }\end{array}$ \\
\hline$\lambda_{42,43}$ & $-0.399 \pm 7.68$ & 5.199 & $\begin{array}{l}P_{W E C S}, \\
Q_{W E C S, P} P_{P V}, \\
Q_{P V}\end{array}$ \\
\hline$\lambda_{45,46}$ & $-1.382 \pm 10.83$ & 14.219 & $\begin{array}{l}P_{P V}, Q_{P V}, \\
P_{W E C S}, Q_{W E C S},\end{array}$ \\
\hline
\end{tabular}

units. When a stable operating point was perturbed, an oscillatory condition emerges until a new stable operating point was attained. However, if system damping was not sufficient to damp the oscillation, critical or even unstable conditions might happen.

Since power contribution from WECS and PV predominantly affected MG stability, the modal analysis was focused on critical modes correlated to output power from those DG units. Non-linear dynamic behaviour of the critical modes should be monitored to evaluate the small signal stability performance of hybrid MG. According to small signal stability analysis in $[18,19]$, The investigation focused on critical modes corresponded to the output power of WECS and PV based DGs which represented as $\lambda 42,43$ and $\lambda 45,46$ respectively. Participation factor analysis suggested that critical modes related to DG unit power output were characterized by low-frequency oscillation as 
Table 6. Distribution and statistical properties of critical modes

\begin{tabular}{|c|c|c|c|c|c|c|c|c|c|c|c|c|}
\hline \multicolumn{13}{|c|}{ Location 1} \\
\hline \multirow{3}{*}{ Period } & \multicolumn{6}{|c|}{$\lambda_{42,43}$} & \multicolumn{6}{|c|}{$\lambda_{45,46}$} \\
\hline & \multicolumn{2}{|c|}{ Real } & \multicolumn{2}{|c|}{ Imaginary } & \multicolumn{2}{|c|}{ Damping } & \multicolumn{2}{|c|}{ Real } & \multicolumn{2}{|c|}{ Imaginary } & \multicolumn{2}{|c|}{ Damping } \\
\hline & Mean & Std & Mean & Std & Mean & Std & Mean & Std & Mean & Std & Mean & Std \\
\hline n-Mar & -1.532 & 0.226 & 10.814 & 0.067 & 14.034 & 2.104 & -0.502 & 0.391 & 6.547 & 1.748 & 6.133 & 20.766 \\
\hline . & -1.527 & & & 0.0 & & & & & 6.697 & & & 9.127 \\
\hline Jul-Sep & -1.616 & 0.148 & 10.789 & 0.039 & 14.818 & 1.374 & -0.506 & 0.216 & 7.094 & 1.071 & .404 & 12.149 \\
\hline ct-Des & -1.421 & 0.226 & 10.842 & 0.072 & 12.992 & 2.103 & -0.587 & 0.273 & 5.975 & 1.865 & 8.837 & 21.8 \\
\hline \multicolumn{13}{|c|}{ Location 2} \\
\hline \multirow{3}{*}{ Period } & \multicolumn{6}{|c|}{$\lambda_{42,43}$} & \multicolumn{6}{|c|}{$\lambda_{45,46}$} \\
\hline & \multicolumn{2}{|c|}{ Real } & \multicolumn{2}{|c|}{ Imaginary } & \multicolumn{2}{|c|}{ Damping } & \multicolumn{2}{|c|}{ Real } & \multicolumn{2}{|c|}{ Imaginary } & \multicolumn{2}{|c|}{ Damping } \\
\hline & Mean & Std & Mean & Std & Mean & Std & Mean & Std & Mean & Std & Mean & Std \\
\hline n-Mar & $\begin{array}{l}-1.393 \\
\end{array}$ & 0.221 & 10.846 & 0.066 & 12.741 & 2.057 & -0.606 & 0.272 & 5.785 & 1.742 & 9.492 & 16.911 \\
\hline & & & 10.845 & 0.072 & 12.598 & & -0.587 & & 5.619 & 1.837 & 8.509 & 20.2 \\
\hline 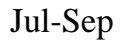 & -1.396 & 0.222 & 10.841 & 0.068 & 12.778 & & -0.604 & 0.262 & 5.789 & 1.754 & 9.373 & 17.88 \\
\hline Dct-Des & -1.278 & 0.209 & 10.883 & 0.076 & 11.674 & 1.963 & -0.561 & 0.435 & 4.887 & 2.078 & 8.058 & 29.813 \\
\hline
\end{tabular}

presented in Table 5. Moreover, it was also monitored that WECS and PV were not fully decoupled, indicated by contributions of active and reactive power output from WECS and PV on critical modes of $\lambda_{42,43}$ and $\lambda_{45,46}$. In base case scenario, both of critical modes were stable with the damping ratio more than 5\%. However, when hybrid MG was operated under fluctuating conditions of wind speed and solar irradiance, the corresponded modes responded randomly and potentially caused instability.

Small signal stability in $\mathrm{MG}$ considering uncertainties of wind speed and solar irradiance was assessed through MCS. Estimated RES distribution functions obtained from the proposed copula method was employed to generate 10000 samples of wind speed and solar irradiance. The generated RES data were then randomly sampled through MC simulation to realize practical scenarios of uncertainties in $\mathrm{MG}$ and determine the system eigenvalues in all possible circumstances of wind speed and irradiance. Under RES variations, the system eigenvalues responded randomly. Hence it was difficult to identify trajectories of the observed modes. Clustering technique such as spectral K-mean can be implemented to extract the investigated modes from system eigenvalues. The proposed clustering method iteratively calculated the affinity matrix of system eigenvalues and determine the eigenvalues cluster based on the Euclidean distance between two eigenvalues. The eigen-clusters were then determined based on the minimum distance between eigenvalues. When a specific cluster of investigated eigenvalues was determined, statistical features regarding the distribution of real and imaginary eigenvalues components, damping ratio and risk of stability can be evaluated. Critical eigenvalues in hybrid MG were characterized by low-frequency oscillation under $2 \mathrm{~Hz}$ as presented in Table 4 . Hence the $\mathrm{K}$-mean clustering technique was applied to cluster the eigenvalues based on the predefined oscillatory frequency of critical modes. The obtained eigen-cluster was then confirmed with participation factor analysis to determine the investigated modes corresponded to output power from RES based DGs in hybrid MG.

Statistical properties and distribution of critical modes from two locations involving the mean and standard deviations of the real part, imaginary part and damping ratio are presented in Table 6. Under wind speed and solar irradiance fluctuations, the critical modes deviated differently. The critical modes corresponded to PV based DG unit $\left(\lambda_{45,46}\right)$ diverged moderately as denoted by low standard deviation of those critical modes. A low standard deviation implied that only short movement of eigenvalues was experienced during fluctuation of irradiance. Conversely, high standard deviations related to critical modes from WECS was monitored, denoted a significant dispersion of those critical modes under wind speed variations. It was suggested that the change in wind speed significantly affected the dynamic response of hybrid MG than the variations of solar irradiance.

Fig. 4 represents samples of critical modes trajectories in a certain season and hour from both areas. The critical modes corresponded to the output power of WECS and PV based DGs were represented as $\lambda_{42,43}$ and $\lambda_{45,46}$ respectively. Variation of solar irradiance influenced the dynamic response of MG considerably. As solar irradiance randomly increased, the eigenvalues of $\lambda_{42,43}$ departed toward the right half 


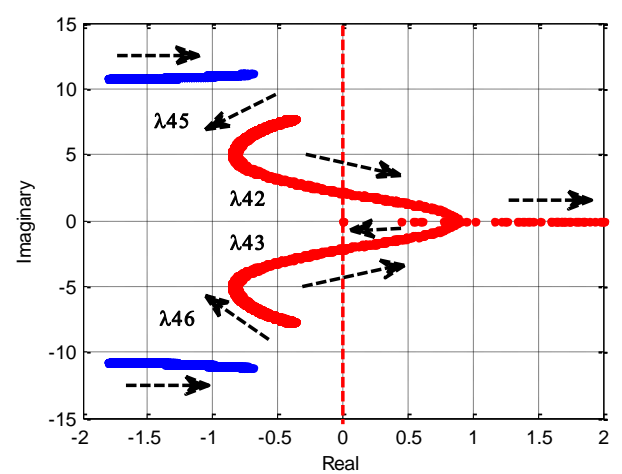

(a)

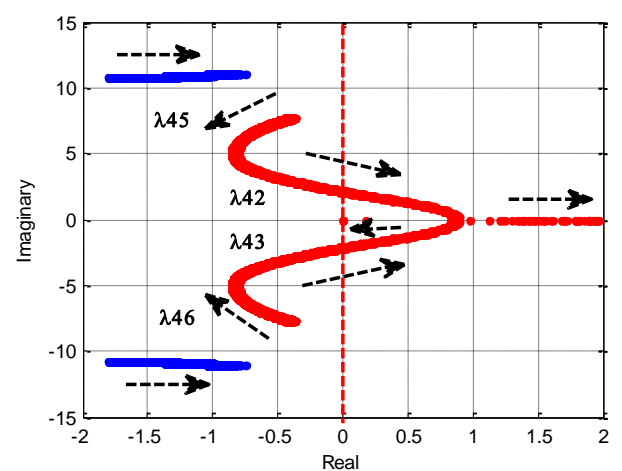

(c)

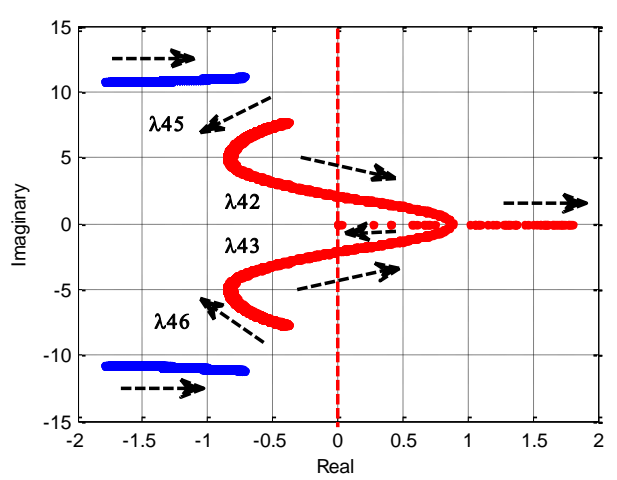

(b)

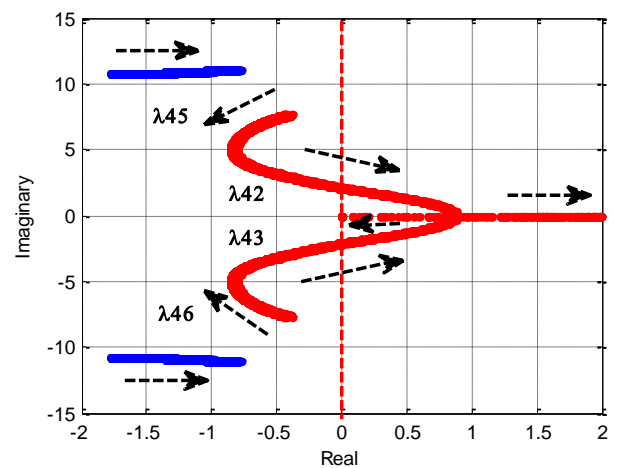

(d)

Figure. 4 Trajectories of sensitive modes from: (a and b) location 1, (c and d) location 2, under RES variations

complex plane, indicated deterioration of small signal stability performance. Even though the less damped situation was monitored under irradiance variations, the corresponded modes relatively stable on the left half side of the complex plane. The system dynamic response suffered when MG was operated under wind speed variations. Sensitive modes of $\lambda_{45,46}$ moved extensively to the right-hand side of the complex plane as wind speed increased. Critical situations with low oscillatory frequency were monitored as sensitive modes departed toward the right-hand side of the complex plane. The unstable situation occurred when the wind speed was more than $15 \mathrm{~m} / \mathrm{s}$. The frequency of WECS modes significantly reduced from $1.21 \mathrm{~Hz}$ to $0.4 \mathrm{~Hz}$ at higher wind speed values. Even in a certain unstable circumstance, the sensitive modes correspond the hybrid MG aligned in a certain point and separated into two real modes. This situation leads to non-oscillatory instability which results in a chaotic situation in MG.

System damping was assessed to evaluate the hybrid MG oscillatory instability risk under the uncertain condition of RES. Assessment of system dynamic response was conducted by evaluating the distribution of damping ratio of the critical mode for every operating state given by random values of wind speed and solar irradiance. Hybrid MG operation can be clustered into three operating states based on the damping condition of the critical modes. The first operating state is a stable MG operation which is related to a well damped dynamic response of islanding hybrid MG. This operating state is characterized by critical modes damping ratio more than $(\zeta \geq 5 \%)$. Second MG operating state is a marginally stable state. In this region, less to the critically damped situation is monitored. The damping ratio of critical modes varied in the range of $0 \%$ to $5 \%(0 \% \leq \zeta \leq 5 \%)$. The undamped oscillation with the continuous increase of oscillatory magnitude is clustered into the third unstable MG operating state. In this region, negative damping ratio $(\zeta<0 \%)$ was observed. Fig. 5 represents cumulative probability distribution of WECS and PV critical modes damping ratio from location 1 and location 2. From critical eigenvalues CDF, it was monitored that when the investigated MG system was subjected to solar irradiance variations, the damping performance can be maintained above the critical limits. A $100 \%$ welldamped system dynamic response was observed, characterized by more than $5 \%$ damping ratio. Conversely, hybrid MG suffered from wind speed uncertainties. The poorly damped oscillatory conditions possibly occurred when the hybrid MG was subjected to wind speed variations. As more fluctuating condition under wind speed variations was experienced, the stability margin decreased 


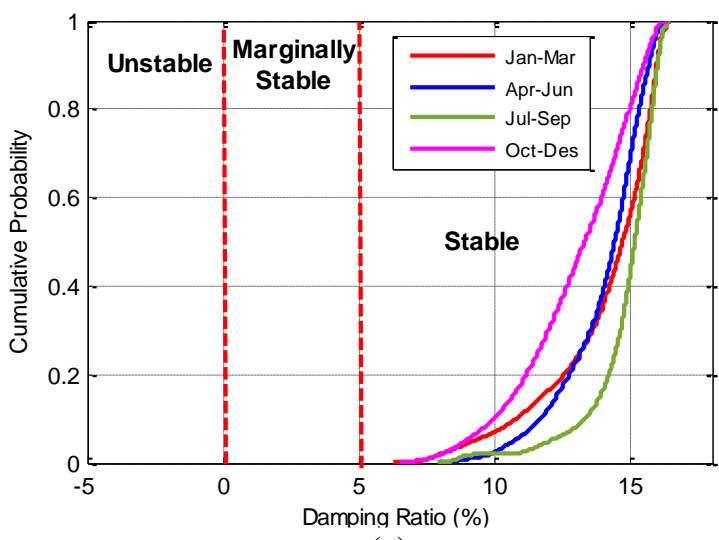

(a)

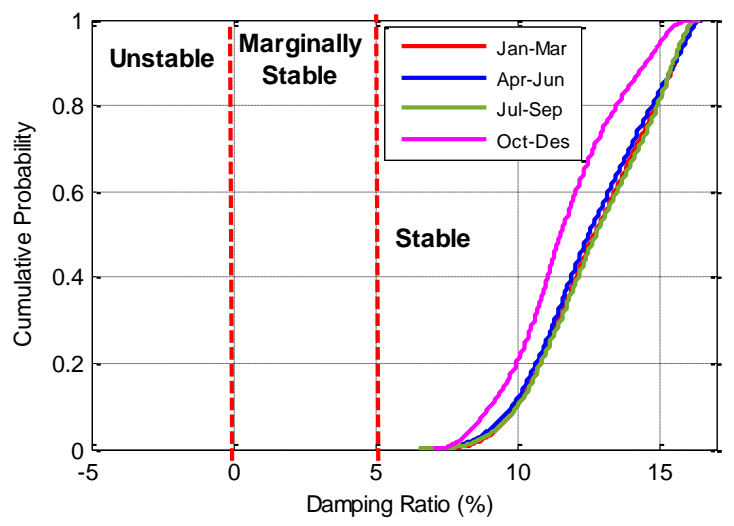

(c)

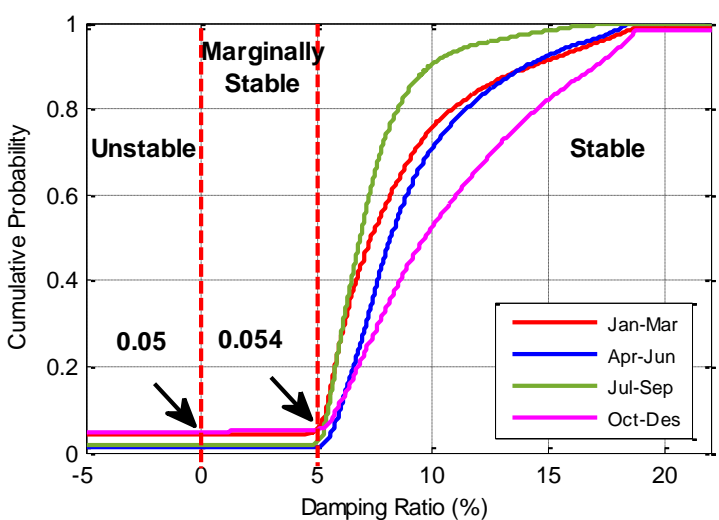

(b)

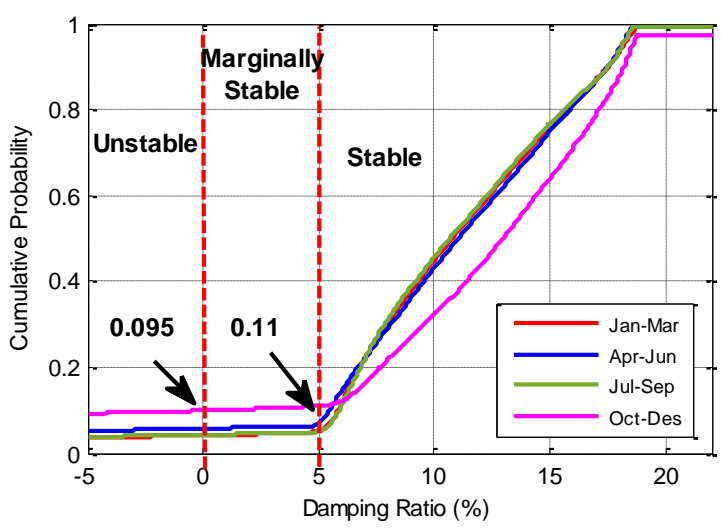

(d)

Figure. 5 CDF of WECS and PV critical modes damping ratio from: (a and b) location 1, (c and d) location 2

considerably. From 10000 generated operating condition samples, a number of critical conditions were identified. In location 1, it was monitored that probability of critical situation with damping ratio in the range of $0 \%$ to $5 \%$ was 0.004 which was equal to 40 critical events. While around 500 or 0.05 probability of unstable events with negative damping took place in location 1. More critical situations were identified in location 2. The 0.095 possibility of instability or 950 unstable events occurred. While around 150 marginally stable situations or 0.015 probability events with damping ratio between $0 \%$ and $5 \%$ were monitored.

\section{Conclusions}

Effects of RES uncertainties in small signal stability performance of islanding operation of hybrid MG is presented in this paper. Student's $t$ - Copula method is applied for estimating the distribution of wind speed and solar irradiance variations, considering their dependencies. It is reported that the proposed estimation method provides more accurate result in estimating the PDF of wind speed and solar irradiance than conventional estimation methods such as Weibull and Gaussian Mixture. The estimated wind speed and solar irradiance from two locations were then applied to hybrid MG system to investigate the small signal stability performance of the investigated MG system under RES changes. From the probabilistic study, it was suggested that the presence of wind speed and solar irradiance uncertainties influence the MG oscillatory stability and dynamic response (This is indicated by the damping value is variated from critical damped (with probability around 0.004), well damped and under damped). Uncertain condition of wind speed introduced more severe effect on system damping than solar irradiation variation, indicated by the extensive movement of the critical eigenvalues toward the imaginary axis.

\section{Conflicts of interest}

"The authors declare no conflict of interest."

\section{Author contributions}

"Conceptualization, Awan Uji Krismanto, Abraham Lomi and N Mithulananthan; methodology, Awan Uji Krismanto, and Herlambang Setiadi; Awan Uji Krismanto, N Mithulananthan and Muhammad Abdillah; validation, Awan Uji 
Krismanto, Abraham Lomi and N Mithulananthan; formal analysis, Awan Uji Krismanto, and Herlambang Setiadi; investigation, Awan Uji Krismanto and Muhammad Abdillah; resources, Awan Uji Krismanto; writing original draft preparation, Awan Uji Krismanto; writing review and editing, Abraham Lomi, and N Mithulananthan; visualization, Awan Uji Krismanto, Muhammad Abdillah, and Herlambang Setiadi. All authors have read and agreed to the published version of the manuscript".

\section{References}

[1] N. Pogaku, M. Prodanovic, and T. C. Green, "Modeling, analysis and testing of autonomous operation of an inverter-based microgrid", IEEE Trans. Power Electron., Vol. 22, No. 2, pp. 613625, 2007, doi: Doi 10.1109/Tpel.2006.890003.

[2] Y. A. R. I. Mohamed and E. F. E. Saadany, "Adaptive Decentralized Droop Controller to Preserve Power Sharing Stability of Paralleled Inverters in Distributed GenerationMicrogrids", IEEE Trans. Power Electron., Vol. 23, No. 6, pp. 2806-2816, 2008.

[3] E. Barklund, N. Pogaku, M. Prodanovic, C. H. Aramburo, and T. C. Green, "Energy Management in Autonomous Microgrid Using Stability-Constrained Droop Control of Inverters", IEEE Trans. Power Electron., Vol. 23, No. 5, pp. 2346-2351, 2008.

[4] H. Ahmadi and H. Seifi, "Probabilistic tuning of Power System Stabilizers considering the wind farm generation uncertainty", Electr. Power Energy Syst., Vol. 63, pp. 565-576, 2014.

[5] J. L. Rueda and D. G. Colome', "Probabilistic performance indexes for small signal stability enhancement in weak wind-hydro-thermal power systems", IET Gener. Transm. Distrib., 2009.

[6] G. L. a, H. Yuea, M. Zhoua, and J. Weib, "Probabilistic assessment of oscillatory stability margin of power systems incorporating wind farms", Electr. Power Energy Syst., Vol. 58, pp. 47-56, 2014.

[7] J. L. Rueda, D. G. Colomé, and I. Erlich, "Assessment and Enhancement of Small Signal Stability Considering Uncertainties", IEEE Trans. Power Syst., Vol. 24, No. 1, 2009.

[8] H. Yue, G. Li, and M. Zhou, "A Probabilistic Approach to Small Signal Stability Analysis of Power Systems with Correlated Wind Sources", J. Electr. Eng. Technol., Vol. 8, pp. 742-751, 2013.

[9] T. R. Ayodele, "Analysis of Monte Carlo
Simulation Sampling Techniques on Small Signal Stability of Wind Generator- Connected Power System", J. Eng. Sci. Technol., Vol. 11, No. 4, pp. 563-583, 2016.

[10] X. Y. Bian, Y. Geng, K. L. Lo, Y. Fu, and Q. B. Zhou, "Coordination of PSSs and SVC Damping Controller to Improve Probabilistic SmallSignal Stability of Power System With Wind Farm Integration", IEEE Trans. Power Syst., Vol. 31, No. 3, 2016.

[11] J. Elizondo, R. Y. Zhang, J. K. White, and J. L. K. Jr., "Robust Small Signal Stability for Microgrids under Uncertainty", Power Electronics for Distributed Generation Systems (PEDG), 2015 IEEE 6th International Symposium on. Aachen, 2015.

[12] X. Xu, T. Lin, and X. Zha, "Probabilistic Analysis of Small Signal Stability of Microgrid Using Point Estimate Method", Sustainable Power Generation and Supply, 2009. SUPERGEN '09. International Conference on. 2009.

[13] M. M. Othman, A. Y. Abdelaziz, Y. G. Hegazi, and W. E. Khattam, "Approach for modelling stochastically dependent renewable energybased generators using diagonal band copula", IET Renew. Power Gener., Vol. 9, No. 7, pp. 809-820, 2015.

[14] P. E. Bett and H. E. Thornton, "The climatological relationships between wind and solar energy supply in Britain", Renew. Energy, Vol. 87, pp. 96-110, 2016.

[15] K. Solbakken, B. Babar, and T. Boström, "Correlation of wind and solar power in highlatitude arctic areas in Northern Norway and Svalbard", Renew. Energy Environ. Sustain, Vol. 1, No. 42, 2016.

[16] J. Munkhammara, J. Widéna, and L. M. Hinkelmanb, "A copula method for simulating correlated instantaneous solar irradiance in spatial networks", Sol. Energy, Vol. 143, pp. 10 21, 2017.

[17] G. Papaefthymiou and D. Kurowicka, "Using Copulas for Modeling Stochastic Dependence in Power System Uncertainty Analysis", IEEE Trans. Power Syst., Vol. 24, No. 1, pp. 40-49, 2009.

[18] A. U. Krismanto, N. Mithulananthan, and O. Krause, "Stability of Renewable Energy based Microgrid in Autonomous Operation", Sustain. Energy, Grids Networks, Vol. 13, pp. 134-147, 2018 , doi: https://doi.org/10.1016/j.segan.2017.12.009.

[19] A. U. Krismanto and M. Nadarajah, "Identification of Modal Interaction and Small 
Signal Stability in Autonomous Microgrid Operation", IET Gen. Transm. Distrib., 2017, doi: 10.1049/iet-gtd.2017.1219.

[20] Y. T. Tan, D. S. Kirschen, and N. Jenkins, “A Model of PV Generation Suitable for Stability Analysis", IEEE Trans. Energy Convers., Vol. 19, No. 4, pp. 748-755, 2004.

[21] C. E. U. Loo, J. B. Ekanayake, and N. Jenkins, "State-Space Modeling of Wind Turbine Generators for Power System Studies", IEEE Trans. Ind. Appl., Vol. 48, pp. 223-232, 2013.

[22] R. B. Nelsen, An Introduction to Copulas. New York: Springer, 2006.

[23] S. Demarta and A. J. McNeil, "The t Copula and Related Copulas", Int. Stat. Rev. / Rev. Int. Stat., Vol. 73, No. 1, pp. 111-129, 2005.

\section{Appendix}

Table 7. List of notations used in this paper

\begin{tabular}{|l|l|}
\hline Symbol & \multicolumn{1}{|c|}{ Meaning } \\
\hline$\Delta P_{\text {meas }}$ & Average active power \\
\hline$\Delta Q_{\text {meas }}$ & Average reactive power \\
\hline$\omega^{*}$ & Frequency reference value \\
\hline$v^{*}$ & Voltage reference value \\
\hline$P_{\text {input }}$ & Actual input \\
\hline$P_{\text {max }}$ & $\begin{array}{l}\text { Maximum power of DG } \\
\text { controller gain }\end{array}$ \\
\hline$m_{\text {max }}$ & $\begin{array}{l}\text { Minimum active droop } \\
\text { controller gain }\end{array}$ \\
\hline$m_{\text {min }}$ & $\begin{array}{l}\text { Solar irradiance reference } \\
\text { value }\end{array}$ \\
\hline$G_{\text {ref }}$ & Sorray \\
\hline$N_{S C}$ & Sumber \\
\hline$N_{p}$ & Array \\
\hline
\end{tabular}

\begin{tabular}{|l|l|}
\hline$n$ & Constant value of PV Arrays \\
\hline$k$ & Constant value of PV Arrays \\
\hline$v_{w 0}$ & $\begin{array}{l}\text { Initial condition of wind } \\
\text { speed }\end{array}$ \\
\hline$C_{\text {opt }}$ & $\begin{array}{l}\text { Efficiency constant of wind } \\
\text { turbine }\end{array}$ \\
\hline$F_{X}$ & CDF of random variable X \\
\hline$F_{y}$ & CDF of random variable Y \\
\hline$v$ & Degree of freedom \\
\hline$\mu$ & $\begin{array}{l}\text { Mean of random variables } \\
\text { or scatter matrix. }\end{array}$ \\
\hline$\Sigma$ & $\begin{array}{l}\text { The correlation-matrix } \\
\text { implied by the dispersion of } \\
\text { matrix } \Sigma\end{array}$ \\
\hline$P$ & $\begin{array}{l}\text { Rank correlation values } \\
\text { density function of the } t \text { - } \\
\text { copula }\end{array}$ \\
\hline$\rho_{r}$ & \\
\hline$C_{v, P}^{t}$ &
\end{tabular}

